\title{
A Technical Modification for Establishing Selective Antegrade Cerebral Perfusion during Pulmonary Endarterectomy
}

\author{
Martin Czerny ${ }^{1}$ Patrick von Samson ${ }^{2}$ Bernward Passlick ${ }^{2}$ Friedhelm Beyersdorf $^{1}$ Johannes Kroll ${ }^{1}$ \\ ${ }^{1}$ University Heart Center Freiburg-Bad Krozingen, Freiburg, Germany \\ 2 University Clinic for Thoracic Surgery, University of Freiburg, \\ Freiburg, Germany \\ Address for correspondence Martin Czerny, MD, MBA, University \\ Heart Center Freiburg- Bad Krozingen, Hugstetterstrasse 55, D-79106 \\ Freiburg, Germany \\ (e-mail: martin.czerny@universitaets-herzzentrum.de).
}

Thorac Cardiovasc Surg Rep 2016;5:33-35.
Abstract
Keywords
- pulmonary arteries
- pulmonary endarterectomy
- selective antegrade cerebral perfusion

\begin{abstract}
Pulmonary endarterectomy (PEA) due to chronic thromboembolic pulmonary hypertension (CTEPH) is mainly performed in deep hypothermic circulatory arrest without additional cerebroprotective means. A 49-year-old man was treated by PEA for CTEPH by using selective antegrade cerebral perfusion by advancing the tip of the systemic perfusion cannula into the brachiocephalic trunk. The postoperative course was uneventful.
\end{abstract}

\section{Introduction}

Selective antegrade cerebral perfusion (SACP) has emerged as a widely used cerebroprotective technique in proximal thoracic aortic surgery. However, the technique is not routinely used in patients undergoing pulmonary endarterectomy (PEA) for chronic thromboembolic pulmonary hypertension (CTEPH). We describe a simple technical modification for easily establishing SACP during PEA.

\section{Technique}

A 49-year-old man was referred for PEA due to CTEPH. The patient was treated by bilateral PEA in hypothermia at $23^{\circ} \mathrm{C}$ with lower body circulatory arrest but continuing cerebral perfusion using SACP via redirection of the systemic arterial inflow cannula (22-French Medtronic EOPA Arterial Cannula, Medtronic Inc., Minneapolis, Minnesota, United States) into the brachiocephalic trunk with consecutive snaring of the vessel and additional occlusion of the left common carotid artery as well the left subclavian artery via vessel loops (-Fig. 1). The ascending aorta was merely clamped for cardioplegia administration. Our protocol includes a flow rate between 7 and $10 \mathrm{~mL} / \mathrm{kg}$ bodyweight per minute with the perfusate temperature equal to the core temperature. Cerebral monitoring is performed via near-infrared spectroscopy using the Somanetics system (Cerebral Oximeter, Somanetics; Troy, Michigan, United States) showing regular cerebral oxygenation patterns during the SACP period. The postoperative course was uneventful.

\section{Comment}

PEA due to CTEPH is mainly performed in deep hypothermic circulatory arrest (DHCA) to provide a bloodless surgical field. Recently, DHCA alone has been shown to be noninferior to DHCA with SACP in patients undergoing PEA. ${ }^{1}$ However, the benefits of SACP in proximal thoracic aortic surgery have been extensively demonstrated. ${ }^{2,3}$ Thereby, SACP in PEA may well present a means to enable higher circulatory arrest temperatures without increasing risk, thereby reducing hypothermic circulatory arrest time and consecutively bleeding complications associated with deep core temperatures and long DHCA times.

The PEACOG trial as well as many other reports on PEA refers to DHCA as a period of DHCA not exceeding 20 minutes interrupted by a period of 10 minutes of reperfusion between DHCA periods. ${ }^{1}$ In other words, antegrade cerebral perfusion
License terms

Stuttgart · New York

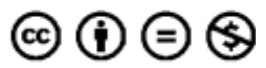

June 24, 2015

accepted after revision

September 15, 2015

published online

May 5, 2016
DOI http://dx.doi.org/

$10.1055 / \mathrm{s}-0035-1566264$. ISSN 2194-7635. 


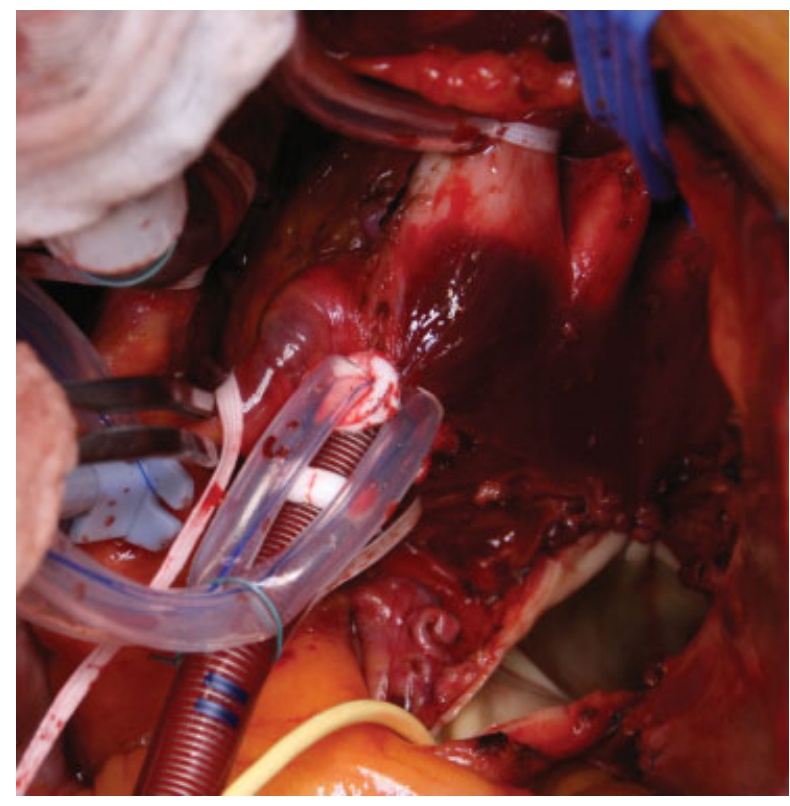

Fig. 1 Intraoperative view depicting the 22-French Medtronic EOPA Arterial Cannula advancing into the brachiocephalic trunk with a snare around the vessel. Furthermore, the left common carotid artery and the left subclavian artery are snared with vessel loops. The left pulmonary artery mainstem is already opened.

(ACP) is used but not in a continuing fashion. This may also well be the reason why the trial did not show any differences regarding cognitive function because DHCA periods always alternated with ACP.
In contrast to proximal thoracic aortic surgery, the ascending aorta and the aortic arch remain unaffected by surgery during PEA except for arterial cannulation. By using a straight cannula as in our case (22-French Medtronic EOPA Arterial Cannula), the tip of the cannula can easily be advanced into the brachiocephalic trunk by tactile control, thereby establishing SACP without additional clamping of the ascending aorta (except for cardioplegia administration) and the distal aortic arch. Without doubt, optimal visibility forms the basis of the surgical result-however, ACP and optimal visibility are not mutually exclusive in our opinion.

Summarizing, this simple technical modification may serve as an adjunct to the armamentarium of the surgeon in performing PEA with SACP to increase the safety margin during systemic circulatory arrest and to decrease DHCA alone-associated collateral injury.

\section{References}

1 Vuylsteke A, Sharples L, Charman G, et al. Circulatory arrest versus cerebral perfusion during pulmonary endarterectomy surgery (PEACOG): a randomised controlled trial. Lancet 2011; 378(9800):1379-1387

2 De Paulis R, Czerny M, Weltert L, et al; EACTS Vascular Domain Group. Current trends in cannulation and neuroprotection during surgery of the aortic arch in Europe. Eur J Cardiothorac Surg 2015; 47(5):917-923

3 Bonser RS, Ranasinghe AM, Loubani M, et al. Evidence, lack of evidence, controversy, and debate in the provision and performance of the surgery of acute type A aortic dissection. J Am Coll Cardiol 2011;58(24):2455-2474 


\section{A Technical Modification for Establishing Selective Antegrade Cerebral Perfusion during Pulmonary Endarterectomy}

\author{
Andreas Boening ${ }^{1}$ Eckhard Mayer ${ }^{2}$ \\ ${ }^{1}$ Department Cardiovascular Surgery, Justus-Liebig-University, \\ Giessen, Germany \\ 2 Department Thoracic Surgery, Thorax Center, Kerckhoff Clinic Bad \\ Nauheim, Bad Nauheim, Germany
}

The better is the enemy of the good.

Progress in medicine has often come from questioning usual practices, aiming toward better solutions for our patients. In the era of "evidence-based medicine," progress and success have to be measurable to show that "better is the enemy of the good." Even in surgery, where eminencebased medicine relying on certain "schools" with traditional therapy forms is still more the rule than the exception, advantages and disadvantages of surgical techniques are subject to clinical studies using elaborate statistical methods for measurement of success of different techniques. During the last years, on-pump and off-pump coronary artery surgery have been compared using these methods, for example (GOPCABE study).

Also for the topic of this case report, namely, the surgical treatment of chronic thromboembolic pulmonary hypertension (CTEPH), there is a study comparing the approach suggested in this report with the standard technique (PEACOG). ${ }^{2}$ The message from this study was that using the standard technique would make surgery easier, while safety was similar to the antegrade cerebral perfusion (ACP) technique.

As the standard technique involves deep hypothermic circulatory arrest (DHCA), it allows for periods without body perfusion of up to 20 minutes, after which a reperfusion is required. This reperfusion is markedly different from ACP, because ACP always requires manipulation of the head vessels with its inherent complications. Moreover, continuous ACP is leading to flow through the pulmonary vasculature, again
Address for correspondence Prof. Dr. Andreas Boening, Department Cardiovascular Surgery, Justus-Liebig-University, Giessen, Germany

Prof. Dr. Eckhard Mayer, Department Thoracic Surgery, Thorax Center, Kerckhoff Clinic Bad Nauheim, Bad Nauheim, Germany (e-mail: andreas.boening@chiru.med.uni-giessen.de; thoraxchirurgie@kerckhoff-klinik.de).

leading to reduced visibility and worse exposure of the segmental and subsegmental pulmonary artery branches.

For these reasons, centers that do surgery for CTEPH routinely (which nowadays means $>100$ cases/year) still rely on DHCA. The results of CTEPH surgery with DHCA are predictable, have low complication and mortality rates, and have a completely uneventful neurologic recovery of the patients. ${ }^{3,4}$ There is international consensus that DHCA is the optimal concept for pulmonary endarterectomy surgery. ${ }^{5,6}$ It seems that the "enemy of the good" still remains to be found in CTEPH surgery.

\section{References}

1 Voltaire. A Philosophical Dictionary. Vol. 2;London: W. Dugdale; 1843:117

2 Vuylsteke A, Sharples L, Charman G, et al. Circulatory arrest versus cerebral perfusion during pulmonary endarterectomy surgery (PEACOG): a randomised controlled trial. Lancet 2011;378(9800): 1379-1387

3 Jamieson SW, Kapelanski DP, Sakakibara N, et al. Pulmonary endarterectomy: experience and lessons learned in 1,500 cases. Ann Thorac Surg 2003;76(5):1457-1462, discussion 1462-1464

4 Jamieson SW. Editorial Comment. Eur J Cardiothorac Surg 2006; 30:241-243

$5 \mathrm{Kim} \mathrm{NH}$, Delcroix M, Jenkins DP, et al. Chronic thromboembolic pulmonary hypertension. J Am Coll Cardiol 2013;62(25, Suppl): D92-D99

6 Konstantinides SV, Torbicki A, Agnelli G, et al; Task Force for the Diagnosis and Management of Acute Pulmonary Embolism of the European Society of Cardiology (ESC). 2014 ESC guidelines on the diagnosis and management of acute pulmonary embolism. Eur Heart J 2014;35(43):3033-3069, 3069a-3069k 